OPEN ACCESS

Edited by:

Charles W. Knapp University of Strathclyde,

United Kingdom

Reviewed by:

Christopher Rensing,

Fujian Agriculture and Forestry

University, China

Seánín Marie McCluskey, Microbiotica, United Kingdom

*Correspondence:

Leonardo N. Andrade

leoandrade02es@gmail.com

Specialty section:

This article was submitted to

Antimicrobials, Resistance and

Chemotherapy,

a section of the journal

Frontiers in Microbiology

Received: 18 January 2018

Accepted: 08 March 2018

Published: 23 March 2018

Citation:

Andrade $L N$, Siqueira TES, Martinez $R$ and Darini ALC (2018)

Multidrug-Resistant CTX-M-(15, 9,

2)- and KPC-2-Producing

Enterobacter hormaechei and

Enterobacter asburiae Isolates Possessed a Set of Acquired Heavy

Metal Tolerance Genes Including a Chromosomal sil Operon (for Acquired Silver Resistance).

Front. Microbiol. 9:539.

doi: 10.3389/fmicb.2018.00539

\section{Multidrug-Resistant CTX-M-(15, 9, 2)- and KPC-2-Producing Enterobacter hormaechei and Enterobacter asburiae Isolates Possessed a Set of Acquired Heavy Metal Tolerance Genes Including a Chromosomal sil Operon (for Acquired Silver Resistance)}

\author{
Leonardo N. Andrade ${ }^{1 *}$, Thiago E. S. Siqueira ${ }^{2}$, Roberto Martinez ${ }^{2}$ and \\ Ana Lucia C. Darini ${ }^{1}$ \\ ${ }^{1}$ Faculdade de Ciencias Farmaceuticas de Ribeirao Preto, Universidade de Sao Paulo, Ribeirao Preto, Brazil, ${ }^{2}$ Faculdade de \\ Medicina de Ribeirao Preto, Universidade de Sao Paulo, Ribeirao Preto, Brazil
}

Bacterial resistance to antibiotics is concern in healthcare-associated infections. On the other hand, bacterial tolerance to other antimicrobials, like heavy metals, has been neglected and underestimated in hospital pathogens. Silver has long been used as an antimicrobial agent and it seems to be an important indicator of heavy metal tolerance. To explore this perspective, we searched for the presence of acquired silver resistance genes (sil operon: silE, silS, silR, silC, silF, silB, silA, and silP) and acquired extended-spectrum cephalosporin and carbapenem resistance genes (b/a $\mathrm{C}_{\mathrm{CX}} \mathrm{M}$ and blakPC) in Enterobacter cloacae Complex (EcC) $(n=27)$ and Enterobacter aerogenes $(n=8)$ isolated from inpatients at a general hospital. Moreover, the genetic background of the silA (silver-efflux pump) and the presence of other acquired heavy metal tolerance genes, $p c o D$ (copper-efflux pump), arsB (arsenite-efflux pump), terF (tellurite resistance protein), and merA (mercuric reductase) were also investigated. Outstandingly, 21/27 (78\%) EcC isolates harbored silA gene located in the chromosome. Complete sil operon was found in 19/21 silA-positive EcC isolates. Interestingly, 8/20 (40\%) E. hormaechei and 5/6 (83\%) E. asburiae co-harbored silA $/ p c o D$ genes and blaCTX-M-(15,2,or9) and/or blakPC-2 genes. Frequent occurrences of arsB, terF, and merA genes were detected, especially in silA/pcoD-positive, multidrug-resistant (MDR) and/or CTX-M-producing isolates. Our study showed co-presence of antibiotic and heavy metal tolerance genes in MDR EcC isolates. In our viewpoint, there are few studies regarding to bacterial heavy metal tolerance and we call attention for more investigations and discussion about this issue in different hospital pathogens.

Keywords: silver, copper, ESBL, carbapenemase, Enterobacter cloacae complex species 
Enterobacter species have been found to be among the top five Gram-negative bacilli that cause hospital infections (Mezzatesta et al., 2012; Davin-Regli and Pages, 2015). Given the increasing identification of carbapenem-resistant and thirdgeneration cephalosporin-resistant Enterobacter isolates, these bacteria are recognized as priority one pathogens (i.e., a critical genus within the family Enterobacteriaceae), according to the World Health Organization (http://www.who.int).

Antibiotic resistance has been widely studied in order to control healthcare-associated infections. On the other hand, tolerance to other antimicrobials (non-antibiotic compounds) has been neglected and underestimated in hospital pathogens. Heavy metals are used to treat superficial bacterial infections and to prevent microbial contamination and control proliferation (e.g., silver and copper), and they are also considered food and/or feed contaminants (e.g., mercury and arsenic) and industrial pollution contaminants (e.g., tellurium; Lemire et al., 2013; Hobman and Crossman, 2015).

Silver is the main non-antibiotic substance that has long been used as an antimicrobial agent and it seems to be an important indicator of heavy metal tolerance. The silverbased substances include silver nitrate and, more recently, silver nanoparticles (nanosilver) have been developed. Bacterial metal homeostasis does not require silver to be metabolized. On the contrary, silver is highly toxic to bacteria, causing cell damage via several mechanisms (Maillard and Hartemann, 2012; Barillo and Marx, 2014; Chandrangsu et al., 2017). Acquired silver resistance was reported to involve derepression of the SilCFBA efflux transporter as a consequence of a mutation in an exogenous (acquired) sil operon gene and additional expression of a periplasmic silversequestration protein (Randall et al., 2015), contributing to reduced intracellular accumulation and activity of silver.

sil operon genes (silE, silS, silR silC, silF, silB, silA, ORF105, and $s i l P$ ) code for apparently structural proteins and for a putative two-component regulatory circuit. SilA (efflux pump), SilB (accessory membrane fusion protein), and SilC (outer membrane porin) correspond to the resistance-nodulation-division (RND)type efflux transporter SilCBA (efflux system); SilE and SilF are periplasmic silver-sequestration proteins; SilP is a putative Ptype ATPase transporter; SilR and SilS comprise a putative twocomponent regulatory circuit; and ORF105 has an amino acid sequence that is homologous to a putative copper chaperone known as CopG, thus it codes for the hypothetical protein SilG, as previously described (Randall et al., 2015). Some studies showed that Sil proteins (e.g., SilE, SilP, and SilCBA efflux system) are also involved in copper tolerance, mainly under anaerobic conditions (Silver, 2003; Kim et al., 2011; Mourão et al., 2015, 2016).

Moreover, other acquired genes have been associated with heavy metal tolerance, such as $p c o D$ (copper efflux pump), $\operatorname{ars} B$ (arsenite efflux pump), terF (tellurite resistance protein), and merA (mercuric reductase) (Hobman and Crossman, 2015; Mourão et al., 2016). Thus, the genetic determinants and mechanisms of heavy metal tolerance have been characterized and linked to enzymic detoxification or efflux of metals.
Considering the few molecular and epidemiological data on the distribution of sil operon genes and the genetic background of silver resistance, and, more generally, the few data on acquired heavy metal tolerance genes in hospital pathogens, we searched for the presence of sil operon genes and beta-lactamase-encoding genes in Enterobacter species isolated from inpatients at a general hospital. Moreover, the genetic background of the silA gene and the presence of other heavy metal tolerance genes were also investigated. In addition, Enterobacter cloacae Complex $(\mathrm{EcC})$ species identification and a bacterial population structure analysis were performed.

We investigated $27 \mathrm{EcC}$ and eight Enterobacter aerogenes isolates (both antibiotic-susceptible and -resistant isolates) recovered from non-repeated inpatients, involving different types of clinical samples and different wards at a university hospital in Brazil (December 2014-April 2015). Clonality was assessed by carrying out genomic DNA digestion with XbaI followed by pulsed-field gel electrophoresis (PFGE), and the results were analyzed using the Tenover criteria (Tenover, 2001) to determine the pulsotypes. EcC species assignment was performed by $h s p 60$ partial gene sequencing (Hoffmann and Roggenkamp, 2003). Multidrug-resistance (MDR) phenotype was defined as nonsusceptibility to at least one agent from three or more antibiotic categories (Magiorakos et al., 2012). sil operon genes (silE, silS, silR, silC, silF, silB, silA, and silP) as well as other genes for heavy metal tolerance ( $p c o D$, ars $B, \operatorname{ter} F$, and mer $A$ ) were investigated by PCR (Woods et al., 2009; Kremer and Hoffmann, 2012; Randall et al., 2015; Mourão et al., 2016). The localization of the silA gene was investigated by carrying out genomic DNA digestion with $S 1$ and I-CEU-I-nuclease followed by PFGE, southern blot and hybridization (Ferreira et al., 2014). Genes coding for ESBLs (bla $a_{\mathrm{CTX}}-\mathrm{M}$, TEM, SHV) and carbapenemases (bla $a_{\mathrm{KPC}}$ IMP, VIM, NDM, OXA-48) were also searched for using PCR and sequencing (Woodford et al., 2006; Bogaerts et al., 2013).

Among the $27 \mathrm{EcC}$ isolates, 20 (74\%; 20 pulsotypes) were identified as Enterobacter hormaechei, six (22\%; 5 pulsotypes) as Enterobacter asburiae, and just one (4\%) as E. cloacae (Table 1). Outstandingly, 21/27 (78\%) EcC isolates harbored silA gene. Notably, silA and pcoD genes were found in $14 / 20$ (70\%) E. hormaechei and all E. asburiae isolates. Only a single E. cloacae isolate (which was silA positive) had no pcoD gene. Moreover, some EcC isolates also carried $\operatorname{ars} B(n=11)$ and/or terF $(n=10)$ and/or merA $(n=6)$ genes; all of them were silA/pcoD positive, except for one E. hormaechei (E04) isolate that was silA/pcoD negative and carried only terF and merA genes. The E. aerogenes isolates did not possess the heavy metal resistance genes investigated (Table $\mathbf{1}$ ).

Complete sil operon genes were found in 19/21 silA-positive $\mathrm{EcC}$ isolates. In the other two isolates, one, E. hormaechei (E21), did not possess the silB gene, and the other, E. cloacae (E48), only possessed the silA gene. Curiously, silC and silR were detected in all the E. aerogenes isolates (all of which were silA negative). All silA-positive EcC isolates possessed the silA gene in the chromosome, although most bacteria studied harbored plasmids, as visualized by $S 1-\mathrm{PFGE}$.

Interestingly, $8 / 20(40 \%) \quad$ E. hormaechei and 5/6 $(83 \%)$ E. asburiae co-harbored silA/pcoD genes and 
TABLE 1 | Heavy metal resistance genes, bla genes, and antibiotic-resistance phenotypes of the Enterobacter spp. isolates studied.

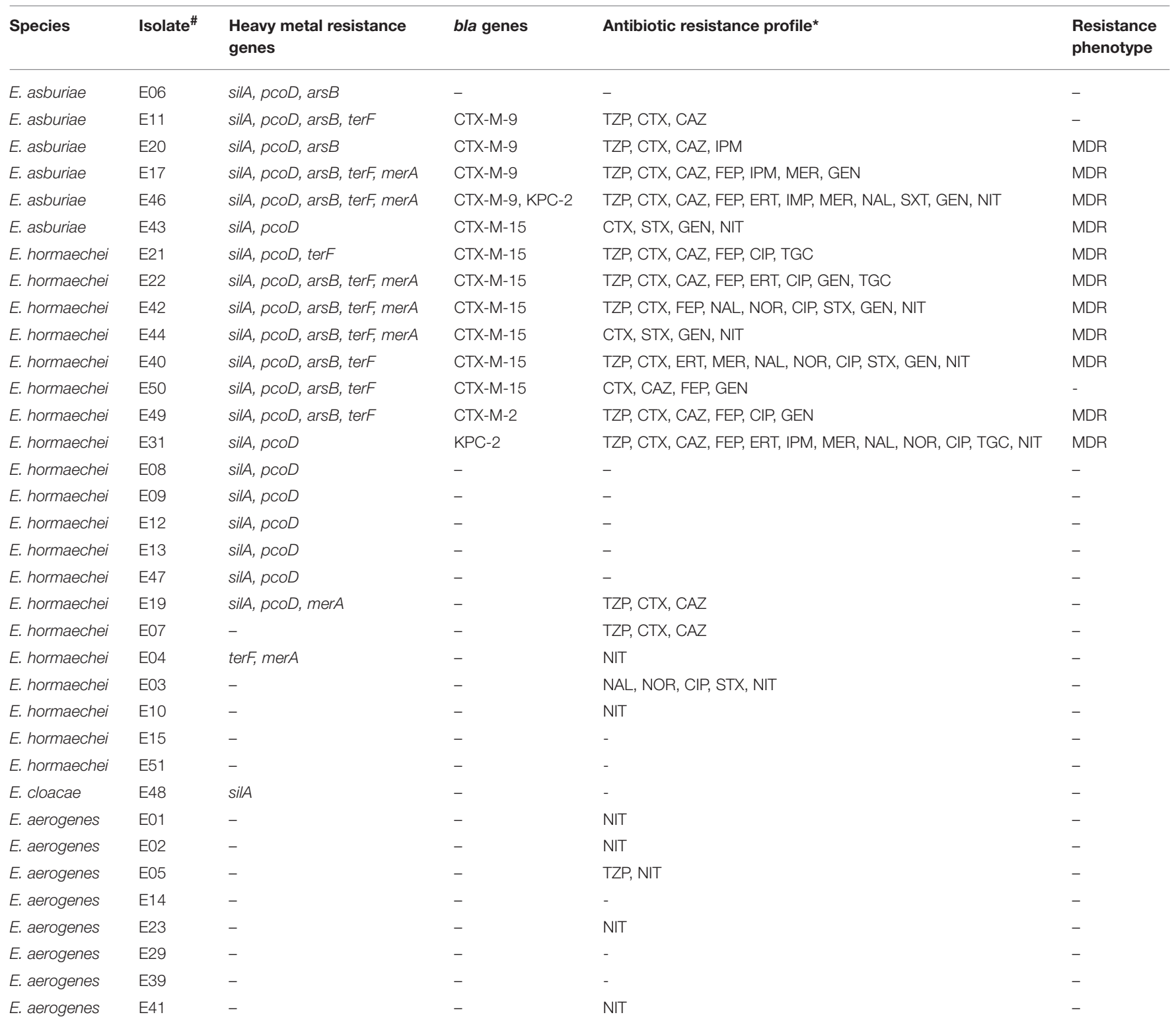

\#E. asburiae E11 and E17 isolates belonged to same pulsotype. The other EcC isolates and E. aerogenes isolates corresponded to different pulsotypes; E. hormaechei E07 and E19 isolates displayed cefotaxime and ceftazidime resistance due to overproducing AmpC (ESBL negative) (data not shown).

*Piperacillin-tazobactam (TZP), cefotaxime (CTX), ceftazidime (CAZ), cefepime (FEP), ertapenem (ERT), imipenem (IPM), meropenem (MER), ertapenem (ERT), gentamicin (GEN), nitrofurantoin (NIT), nalidixic acid (NAL), norfloxacin (NOR), ciprofloxacin (CIP), trimethoprim-sulfamethoxazole (SXT), tigecycline (TGC).

MDR, multidrug-resistance (Magiorakos et al., 2012). For the determination of the MDR phenotype, antibiotic intrinsic resistance (data not shown) and antibiotic therapeutic concentrations achieved only in urine (e.g., in the case of nitrofurantoin) were excluded; Enterobacter cloacae Complex (EcC) species and Enterobacter aerogenes are intrinsically resistant to ampicillin, ampicillin-sulbactam, amoxicillin-clavulanate, first-generation cephalosporin (cefazolin and cephalothin), second-generation cephalosporin (cefuroxime), and cephamycins (cefoxitin and cefotetan).

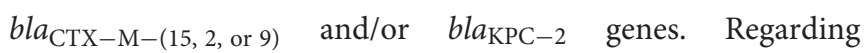
the MDR phenotype, $7 / 20(35 \%)$ E. hormaechei and $4 / 6$ $(67 \%)$ E. asburiae were MDR, amounting to a total of $11 / 26$ EcC isolates that were silA positive and CTX-M and/or Klebsiella pneumoniae Carbapenemase (KPC) producers. Nevertheless, the single E. cloacae and the E. aerogenes isolates neither possessed acquired bla genes nor the MDR phenotype (Table 1).
E. hormaechei and E. asburiae were the most frequently identified $\mathrm{EcC}$ species and they appear to be common $\mathrm{EcC}$ species isolated from human infections, representing a concern because there are few data on these species (Mezzatesta et al., 2012). The high diversity of pulsotypes among the E. hormaechei and E. asburiae isolates as well as the high occurrence of the silA gene in these species suggest non-clonal spread and long-term silver resistance among these species at the hospital studied. 
EcC species appear to be important sil-carrying bacteria compared to other Gram-negative bacilli (Woods et al., 2009; Sütterlin et al., 2012). A silver-resistant E. cloacae strain was previously detected with several sil operon genes (silE, silP, and silS) but no silA gene (potentially due to it not being searched for; Sütterlin et al., 2012; Finley et al., 2015). The silver-resistance phenotype in $\mathrm{EcC}$ isolates might also occur due to derepression of the endogenous cus operon and loss of porins, as characterized previously (Randall et al., 2015).

The sil operon has been reported to be inducible in the presence of silver (Randall et al., 2015), which also explains the non-direct phenotypic resistance among isolates carrying sil genes. Thus, the inducible expression of silver resistance could contribute to the selection for silver-resistant pathogens, mainly among bacteria carrying the complete sil operon and overexpressing the SilCFBA efflux transporter (Randall et al., 2015).

The chromosomal silA location indicates that silA provides an advantageous trait, as this genetic determinant of silver resistance was detected in multiple clones of E. hormaechei and E. asburiae and in a single E. cloacae isolate. Chromosomal integration of acquired antimicrobial-resistance genes, commonly plasmidmediated, has been reported in laboratory and in silico studies; the chromosomal integration is likely explained by genetic uptake and chromosomal recombination/integration. In addition, plasmids harboring silA (and/or $p c o D$ ) genes have also been detected, mainly in Salmonella (Ferreira et al., 2014; Fang et al., 2016; Mourão et al., 2016).

The $p c o D$ gene (along with the silA gene) was very common in E. hormaechei and E. asburiae isolates and it also seems to be a frequently acquired heavy metal resistance gene in these $\mathrm{EcC}$ species. Copper is essential for bacterial metabolism at normal cellular concentrations. Nevertheless, at toxic concentrations, copper causes damage in bacterial cells by several different mechanisms of action (Lemire et al., 2013; Hobman and Crossman, 2015; Staehlin et al., 2016; Chandrangsu et al., 2017). In addition to the obvious contribution to copper tolerance, pcoD (and silA) genes have been related to the emergence of specific clinically relevant MDR Salmonella serotypes/clones, highlighting the relevance of copper tolerance in anaerobic conditions (Mourão et al., 2016). In addition, the pcoD operon and copper efflux has been shown to be associated with bacterial survival in amoebae, and copper-resistance determinants have been reported to be located in a "copper pathogenicity island," contributing to the selection of copper-resistant bacteria. The pco operon has frequently been found to be encoded adjacent to the sil operon, contributing to heavy metal resistance co-selection (Woods et al., 2009; Hao et al., 2015, 2016; Pal et al., 2015).

Interestingly, the acquired heavy metal resistance genes $\operatorname{ars} B, \operatorname{ter} F$, and merA were also detected in the E. hormaechei and E. asburiae isolates, particularly in the silA/pcoD-positive, MDR and/or CTX-M-producing isolates. Several interesting associations involving theses heavy metal resistance genes have been demonstrated. For example, the ars $B$ gene is more related to Salmonella enterica serovar Kentucky than other serovars isolated from poultry and the ars, sil, and pco operons are co-carried on epidemic plasmids (e.g., IncFII $_{\mathrm{K} 1}$ ) in MDR
K. pneumoniae clones (Joerger et al., 2010; Sandegren et al., 2012; Chen et al., 2013; Chen and Rosen, 2014). In addition, tellurite resistance (ter $W$ gene, a ter operon gene) is commonly reported in community hypervirulent $K$. pneumoniae clonal groups (Taylor, 1999; Passet and Brisse, 2015), and mer operon genes are associated with many Tn21-like transposons that usually co-harbor antibiotic-resistance genes (Boyd and Barkay, 2012). Thus, arsenic, tellurium, mercury, as well as copper tolerance and/or other advantageous characteristics, need further investigation in Enterobacter species and other hospital pathogens.

The selection of antibiotic-resistant bacteria has been amplified by co-resistance, that is, the presence of different resistance mechanisms (encoded by mutated or acquired genes) affecting different antimicrobial classes (Cantón and RuizGarbajosa, 2011; Wong et al., 2014; Kumar et al., 2016). We demonstrated that the CTX-M- and/or KPC-producing EcC isolates studied here (most of which were MDR bacteria) possessed a larger set of heavy metal tolerance genes (all of which were silA/pcoD positive and most of which were also arsB, terF, and merA positive) than the non-acquired beta-lactamase producers, indicating co-resistance involving antibiotics and heavy metals and suggesting co-selection of silver/copper- and acquired cephalosporin/carbapenem-resistance genes. Likewise, the silA/pcoD-negative results seem to be more associated with $E$. aerogenes than $\mathrm{EcC}$ species (Table 1). There was a close association between the bla $a_{\mathrm{CTX}-\mathrm{M}}$ gene and the silA (and $p c o D$ ) genes among the isolates studied here, and this finding (silver-resistance genes and/or silver-resistance phenotype) was previously reported for clinical Escherichia coli isolates (Sütterlin et al., 2012, 2014; Deus et al., 2017). Moreover, there have been reports of silver-resistant (and/or silA-positive) isolates associated with silver minimal inhibitory concentrations that could impact the management of non-invasive bacterial infections (such as those found among burn patients; Finley et al., 2015; Deus et al., 2017).

Notably, MDR bacteria are a worldwide problem and have caused antibiotic failure, so new alternative antibiotics and treatment approaches have been studied (Dizaj et al., 2014; de Oliveira et al., 2017; Wang et al., 2017). The combined action of nanosilver and antibiotics has been shown to have additive or synergistic antibacterial action, including the ability to restore the bactericidal activity of inactive antibiotics against MDR Enterobacteriaceae and Pseudomonas aeruginosa (Panácek et al., 2015, 2016; Salomoni et al., 2017). However, the versatility and practicality of nanosilver treatment for bacteria that are coresistant to antibiotics and silver should be investigated in future studies, including comparison with soluble silver salts. For this purpose, it is essential to know the epidemiology of the genetic determinants of silver resistance and to understand the molecular mechanisms of silver resistance in different hospital pathogens. Our study contributes to this understanding, and it characterized acquired silver-resistance genes in clinically important bacterial species that were isolated from multiple inpatients, clinical samples, and wards at a general hospital.

Considering the increasing use of silver in medical devices (e.g., catheters and dressings) and consumer household products 
(e.g., antiperspirants and clothes), and also based on the dramatic increase in reports of silver-resistant bacteria, silver resistance should be a concern beyond the silver resistance seen in isolates infecting burn patients (Lemire et al., 2013; Marx and Barillo, 2014; Hobman and Crossman, 2015).

In summary, our study indicates the common occurrence of acquired sil operon genes and the $p c o D$ gene exclusively in the EcC species, which display high clonal diversity. The study highlights the presence of the silA gene in the chromosome, indicating that it gives rise to an advantageous and adaptive trait. Frequent occurrences of $\operatorname{ars} B, \operatorname{ter} F$, and mer $A$ genes were also detected, especially in silA/pcoD-positive, MDR and/or CTX-Mproducing isolates. The co-presence of acquired silver/copperresistance (silA/pcoD) and cephalosporin/carbapenem-resistance $\left(b l a_{\mathrm{CTX}-\mathrm{M}}\right.$ and $\left.b l a_{\mathrm{KPC}-2}\right)$ genes in the MDR EcC isolates was noteworthy.

Due to absence of surveillance and laboratory testing, bacterial heavy metal resistance can silently boost the antibiotic resistance contributing to selection and spreading of MDR bacteria. In our viewpoint, there are few studies regarding to bacterial heavy metal tolerance and we call attention to more investigations and discussion about this issue, toward detection and monitoring of this neglected and underestimated problem in different hospital pathogens.

\section{REFERENCES}

Barillo, D. J., and Marx, D. E. (2014). Silver in medicine: a brief history BC 335 to present. Burns 40(Suppl. 1), S3-S8. doi: 10.1016/j.burns.2014.09.009

Bogaerts, P., Rezende De Castro, R., De Mendonca, R., Huang, T. D., Denis, O., and Glupczynski, Y. (2013). Validation of carbapenemase and extended-spectrum beta-lactamase multiplex endpoint PCR assays according to ISO 15189. J. Antimicrob. Chemother. 68, 1576-1582. doi: 10.1093/jac/dkt065

Boyd, E. S., and Barkay, T. (2012). The mercury resistance operon: from an origin in a geothermal environment to an efficient detoxification machine. Front. Microbiol. 3:349. doi: 10.3389/fmicb.2012.00349

Cantón, R., and Ruiz-Garbajosa, P. (2011). Co-resistance: an opportunity for the bacteria and resistance genes. Curr. Opin. Pharmacol. 11, 477-485. doi: 10.1016/j.coph.2011.07.007

Chandrangsu, P., Rensing, C., and Helmann, J. D. (2017). Metal homeostasis and resistance in bacteria. Nat. Rev. Microbiol. 15, 338-350. doi: 10.1038/nrmicro.2017.15

Chen, J., and Rosen, B. P. (2014). Biosensors for inorganic and organic arsenicals. Biosensors 4, 494-512. doi: 10.3390/bios4040494

Chen, L., Chavda, K. D., Melano, R. G., Jacobs, M. R., Levi, M. H., Bonomo, R. A., et al. (2013). Complete sequence of a bla(KPC-2)-harboring IncFII(K1) plasmid from a Klebsiella pneumoniae sequence type 258 strain. Antimicrob. Agents Chemother. 57, 1542-1545. doi: 10.1128/AAC.02332-12

Davin-Regli, A., and Pages, J. M. (2015). Enterobacter aerogenes and Enterobacter cloacae; versatile bacterial pathogens confronting antibiotic treatment. Front. Microbiol. 6:392. doi: 10.3389/fmicb.2015.00392

de Oliveira, J. F. A., Saito, A., Bido, A. T., Kobarg, J., Stassen, H. K., and Cardoso, M. B. (2017). Defeating bacterial resistance and preventing mammalian cells toxicity through rational design of antibiotic-functionalized nanoparticles. Sci. Rep. 7:1326. doi: 10.1038/s41598-017-01209-1

Deus, D., Krischek, C., Pfeifer, Y., Sharifi, A. R., Fiegen, U., Reich, F., et al. (2017). Comparative analysis of the susceptibility to biocides and heavy metals of extended-spectrum beta-lactamase-producing Escherichia coli isolates of human and avian origin, Germany. Diagn. Microbiol. Infect. Dis. 88, 88-92. doi: 10.1016/j.diagmicrobio.2017.01.023

\section{AUTHOR CONTRIBUTIONS}

LA was responsible for the study, and performed all the molecular characterizations of the bacteria and the antibiotic- and heavy metal resistance genes. TS and RM carried out the microbiological investigation of the bacterial isolates. $\mathrm{LA}$ and $\mathrm{AD}$ coordinated the research and wrote the manuscript.

\section{FUNDING}

Research grant 2014/14494-8, São Paulo Research Foundation (FAPESP). LA was supported by a postdoctoral fellowship from Coordination for the Improvement of the Higher Education Personnel (CAPES), Programa Nacional de Pós Doutorado (PNPD)/CAPES 2015.

\section{ACKNOWLEDGMENTS}

We are grateful for the technical support of the staff at the Microbiology Laboratory at the Hospital das Clinicas of Ribeirao Preto Medical School. We are also thankful for the technical support of Natalia Columbaro Moreira (undergraduate fellowship FAPESP 2016/09150-3).

Dizaj, S. M., Lotfipour, F., Barzegar-Jalali, M., Zarrintan, M. H., and Adibkia, K. (2014). Antimicrobial activity of the metals and metal oxide nanoparticles. Mater. Sci. Eng. C Mater. Biol. Appl. 44, 278-284. doi: 10.1016/j.msec.2014.08.031

Fang, L., Li, X., Li, L., Li, S., Liao, X., Sun, J., et al. (2016). Co-spread of metal and antibiotic resistance within ST3-IncHI2 plasmids from E. coli isolates of food-producing animals. Sci. Rep. 6:25312. doi: 10.1038/srep25312

Ferreira, J. C., Penha Filho, R. A., Andrade, L. N., Berchieri, A. Jr., and Darini, A. L. (2014). Detection of chromosomal bla(CTX-M-2) in diverse Escherichia coli isolates from healthy broiler chickens. Clin. Microbiol. Infect. 20, O623-O626. doi: 10.1111/1469-0691.12531

Finley, P. J., Norton, R., Austin, C., Mitchell, A., Zank, S., and Durham, P. (2015). unprecedented silver resistance in clinically isolated Enterobacteriaceae: major implications for burn and wound management. Antimicrob. Agents Chemother. 59, 4734-4741. doi: 10.1128/AAC.00026-15

Hao, X., Luthje, F. L., Qin, Y., McDevitt, S. F., Lutay, N., Hobman, J. L., et al. (2015). Survival in amoeba-a major selection pressure on the presence of bacterial copper and zinc resistance determinants? Identification of a “copper pathogenicity island". Appl. Microbiol. Biotechnol. 99, 5817-5824. doi: 10.1007/s00253-015-6749-0

Hao, X., Luthje, F., Ronn, R., German, N. A., Li, X., Huang, F., et al. (2016). A role for copper in protozoan grazing - two billion years selecting for bacterial copper resistance. Mol. Microbiol. 102, 628-641. doi: 10.1111/mmi.13483

Hobman, J. L., and Crossman, L. C. (2015). Bacterial antimicrobial metal ion resistance. J. Med. Microbiol. 64, 471-497. doi: 10.1099/jmm.0.02 3036-0

Hoffmann, H., and Roggenkamp, A. (2003). Population genetics of the nomenspecies Enterobacter cloacae. Appl. Environ. Microbiol. 69, 5306-5318. doi: 10.1128/AEM.69.9.5306-5318.2003

Joerger, R. D., Hanning, I. B., and Ricke, S. C. (2010). Presence of arsenic resistance in Salmonella enterica serovar Kentucky and other serovars isolated from poultry. Avian Dis. 54, 1178-1182. doi: 10.1637/9285-022210-Reg.1

Kim, E. H., Nies, D. H., McEvoy, M. M., and Rensing, C. (2011). Switch or funnel: how RND-type transport systems control periplasmic metal homeostasis. $J$. Bacteriol. 193, 2381-2387. doi: 10.1128/JB.01323-10 
Kremer, A. N., and Hoffmann, H. (2012). Subtractive hybridization yields a silver resistance determinant unique to nosocomial pathogens in the Enterobacter cloacae complex. J. Clin. Microbiol. 50, 3249-3257. doi: 10.1128/JCM.00885-12

Kumar, M., Saha, S., and Subudhi, E. (2016). More furious than ever: Escherichia coli-acquired co-resistance toward colistin and carbapenems. Clin. Infect. Dis. 63, 1267-1268. doi: 10.1093/cid/ciw508

Lemire, J. A., Harrison, J. J., and Turner, R. J. (2013). Antimicrobial activity of metals: mechanisms, molecular targets and applications. Nat. Rev. Microbiol. 11, 371-384. doi: 10.1038/nrmicro3028

Magiorakos, A. P., Srinivasan, A., Carey, R. B., Carmeli, Y., Falagas, M. E., Giske, C. G., et al. (2012). Multidrug-resistant, extensively drug-resistant and pandrug-resistant bacteria: an international expert proposal for interim standard definitions for acquired resistance. Clin. Microbiol. Infect. 18, 268-281. doi: 10.1111/j.1469-0691.2011.03570.x

Maillard, J.-Y., and Hartemann, P. (2012). Silver as an antimicrobial: facts and gaps in knowledge. Crit. Rev. Microbiol. 39, 373-383. doi: 10.3109/1040841X.2012.713323

Marx, D. E., and Barillo, D. J. (2014). Silver in medicine: the basic science. Burns 40 (Suppl. 1), S9-S18. doi: 10.1016/j.burns.2014.09.010

Mezzatesta, M. L., Gona, F., and Stefani, S. (2012). Enterobacter cloacae complex: clinical impact and emerging antibiotic resistance. Future Microbiol. 7, 887-902. doi: 10.2217/fmb.12.61

Mourão, J., Marcal, S., Ramos, P., Campos, J., Machado, J., Peixe, L., et al. (2016). Tolerance to multiple metal stressors in emerging non-typhoidal MDR Salmonella serotypes: a relevant role for copper in anaerobic conditions. J. Antimicrob. Chemother. 71, 2147-2157. doi: 10.1093/jac/dkw120

Mourão, J., Novais, C., Machado, J., Peixe, L., and Antunes, P. (2015). Metal tolerance in emerging clinically relevant multidrug-resistant Salmonella enterica serotype 4,[5],12:i:- clones circulating in Europe. Int. J. Antimicrob. Agents 45, 610-616. doi: 10.1016/j.ijantimicag.2015.01.013

Pal, C., Bengtsson-Palme, J., Kristiansson, E., and Larsson, D. G. (2015). Cooccurrence of resistance genes to antibiotics, biocides and metals reveals novel insights into their co-selection potential. BMC Genomics 16:964. doi: 10.1186/s12864-015-2153-5

Panácek, A., Smekalova, M., Kilianova, M., Prucek, R., Bogdanova, K., Vecerova, R., et al. (2015). Strong and nonspecific synergistic antibacterial efficiency of antibiotics combined with silver nanoparticles at very low concentrations showing no cytotoxic effect. Molecules 21:E26. doi: 10.3390/molecules21010026

Panácek, A., Smekalova, M., Vecerova, R., Bogdanova, K., Roderova, M., Kolar, M., et al. (2016). Silver nanoparticles strongly enhance and restore bactericidal activity of inactive antibiotics against multiresistant Enterobacteriaceae. Colloids Surf. B Biointerfaces 142, 392-399. doi: 10.1016/j.colsurfb.2016.03.007

Passet, V., and Brisse, S. (2015). Association of tellurite resistance with hypervirulent clonal groups of Klebsiella pneumoniae. J. Clin. Microbiol. 53, 1380-1382. doi: 10.1128/JCM.03053-14

Randall, C. P., Gupta, A., Jackson, N., Busse, D., and O’neill, A. J. (2015). Silver resistance in Gram-negative bacteria: a dissection of endogenous and exogenous mechanisms. J. Antimicrob. Chemother. 70, 1037-1046. doi: $10.1093 / \mathrm{jac} / \mathrm{dku} 523$

Salomoni, R., Leo, P., Montemor, A. F., Rinaldi, B. G., and Rodrigues, M. (2017). Antibacterial effect of silver nanoparticles in Pseudomonas aeruginosa. Nanotechnol. Sci. Appl. 10, 115-121. doi: 10.2147/NSA.S133415
Sandegren, L., Linkevicius, M., Lytsy, B., Melhus, A., and Andersson, D. I. (2012). Transfer of an Escherichia coli ST131 multiresistance cassette has created a Klebsiella pneumoniae-specific plasmid associated with a major nosocomial outbreak. J. Antimicrob. Chemother. 67, 74-83. doi: 10.1093/jac/dkr405

Silver, S. (2003). Bacterial silver resistance: molecular biology and uses and misuses of silver compounds. FEMS Microbiol. Rev. 27, 341-353. doi: 10.1016/S0168-6445(03)00047-0

Staehlin, B. M., Gibbons, J. G., Rokas, A., O’halloran, T. V., and Slot, J. C. (2016). Evolution of a heavy metal homeostasis/resistance island reflects increasing copper stress in Enterobacteria. Genome Biol. Evol. 8, 811-826. doi: 10.1093/gbe/evw031

Sütterlin, S., Edquist, P., Sandegren, L., Adler, M., Tangden, T., Drobni, M., et al. (2014). Silver resistance genes are overrepresented among Escherichia coli isolates with CTX-M production. Appl. Environ. Microbiol. 80, 6863-6869. doi: 10.1128/AEM.01803-14

Sütterlin, S., Tano, E., Bergsten, A., Tallberg, A. B., and Melhus, A. (2012). Effects of silver-based wound dressings on the bacterial flora in chronic leg ulcers and its susceptibility in vitro to silver. Acta Derm. Venereol. 92, 34-39. doi: 10.2340/00015555-1170

Taylor, D. E. (1999). Bacterial tellurite resistance. Trends Microbiol. 7, 111-115. doi: 10.1016/S0966-842X(99)01454-7

Tenover, F. C. (2001). Development and spread of bacterial resistance to antimicrobial agents: an overview. Clin. Infect. Dis. 33(Suppl. 3), S108-S115. doi: $10.1086 / 321834$

Wang, L., Hu, C., and Shao, L. (2017). The antimicrobial activity of nanoparticles: present situation and prospects for the future. Int. J. Nanomedicine 12, 1227-1249. doi: 10.2147/IJN.S121956

Wong, P. H., Von Krosigk, M., Roscoe, D. L., Lau, T. T., Yousefi, M., and Bowie, W. R. (2014). Antimicrobial co-resistance patterns of gram-negative bacilli isolated from bloodstream infections: a longitudinal epidemiological study from 2002-2011. BMC Infect. Dis. 14:393. doi: 10.1186/1471-2334-1 4-393

Woodford, N., Fagan, E. J., and Ellington, M. J. (2006). Multiplex PCR for rapid detection of genes encoding CTX-M extended-spectrum (beta)lactamases. J. Antimicrob. Chemother. 57, 154-155. doi: 10.1093/jac/ dki412

Woods, E. J., Cochrane, C. A., and Percival, S. L. (2009). Prevalence of silver resistance genes in bacteria isolated from human and horse wounds. Vet. Microbiol. 138, 325-329. doi: 10.1016/j.vetmic.2009.0 3.023

Conflict of Interest Statement: The authors declare that the research was conducted in the absence of any commercial or financial relationships that could be construed as a potential conflict of interest.

Copyright (c) 2018 Andrade, Siqueira, Martinez and Darini. This is an open-access article distributed under the terms of the Creative Commons Attribution License (CC $B Y)$. The use, distribution or reproduction in other forums is permitted, provided the original author(s) and the copyright owner are credited and that the original publication in this journal is cited, in accordance with accepted academic practice. No use, distribution or reproduction is permitted which does not comply with these terms. 